\title{
Cosine-Gauss Plasmon Beam: A Localized Long- Range Nondiffracting Surface Wave
}

\section{Citation}

Lin, Jiao, Jean Dellinger, Patrice Genevet, Benoit Cluzel, Frederique de Fornel, and Federico Capasso. 2012. "Cosine-Gauss Plasmon Beam: A Localized Long-Range Nondiffracting Surface Wave." Physical Review Letters 109 (9). https://doi.org/10.1103/physrevlett.109.093904.

\section{Permanent link}

http://nrs.harvard.edu/urn-3:HUL.InstRepos:41371363

\section{Terms of Use}

This article was downloaded from Harvard University's DASH repository, and is made available under the terms and conditions applicable to Other Posted Material, as set forth at http:// nrs.harvard.edu/urn-3:HUL.InstRepos:dash.current.terms-of-use\#LAA

\section{Share Your Story}

The Harvard community has made this article openly available.

Please share how this access benefits you. Submit a story.

Accessibility 


\title{
Cosine-Gauss Plasmon Beam: A Localized Long-Range Nondiffracting Surface Wave
}

\author{
Jiao Lin, ${ }^{1,2}$ Jean Dellinger, ${ }^{3}$ Patrice Genevet, ${ }^{1,4}$ Benoit Cluzel, ${ }^{3}$ Frederique de Fornel, ${ }^{3}$ and Federico Capasso ${ }^{1, *}$ \\ ${ }^{1}$ School of Engineering and Applied Sciences, Harvard University, Cambridge, Massachusetts 02138, USA \\ ${ }^{2}$ Singapore Institute of Manufacturing Technology, 71 Nanyang Avenue, Singapore 638075, Singapore \\ ${ }^{3}$ Laboratoire Interdisciplinaire Carnot de Bourgogne, UMR CNRS 6303, 9 avenue Savary, Dijon, 21078, France \\ ${ }^{4}$ Institute for Quantum Studies and Department of Physics, Texas A\&M University, College Station, Texas 77843, USA
}

(Received 5 May 2012; published 31 August 2012)

\begin{abstract}
A new surface wave is introduced, the cosine-Gauss beam, which does not diffract while it propagates in a straight line and tightly bound to the metallic surface for distances up to $80 \mu \mathrm{m}$. The generation of this highly localized wave is shown to be straightforward and highly controllable, with varying degrees of transverse confinement and directionality, by fabricating a plasmon launcher consisting of intersecting metallic gratings. Cosine-Gauss beams have potential for applications in plasmonics, notably for efficient coupling to nanophotonic devices, opening up new design possibilities for next-generation optical interconnects.
\end{abstract}

DOI: 10.1103/PhysRevLett.109.093904

PACS numbers: 42.25.Fx, 03.50.De, 41.20.- $\mathrm{q}$

The expansion of the transverse dimension of a propagating wave, a phenomenon originating from diffraction, is ubiquitous for all kinds of waves including sound, electromagnetic, and even matter waves. In optics, several techniques have been proposed to counterbalance this effect, for example, by considering the propagation of intense light beams in an optical medium with nonlinearities [1]. In the linear regime, the family of so-called diffraction-free beams has long been an active research area since the introduction of the Bessel beam [2,3], a solution of the Helmholtz equation, which has a transverse intensity distribution independent of the propagation distance. Bessel beams were followed by the discovery of other nondiffracting solutions [4,5] including the recent free-space Airy beams [6,7]. However, very little effort has been devoted to constructing diffraction-free surface waves such as surface plasmon polaritons (SPPs) that are propagating electromagnetic surface waves tightly bound to a metaldielectric interface. The strong subwavelength confinement of light offered by SPPs has generated considerable interest in plasmonics as a bridge between electronics and photonics for applications such as on-chip interconnects in the next generation of photonics circuits [8-10]. The propagation of SPPs in general suffers from a high attenuation due to a combination of dissipation (Ohmic loss) and radiative losses associated with interface roughness. Various methods have been proposed to increase their propagation length [11-13]. Because of their wave nature, SPPs also undergo diffraction in the plane of the interface, which is an additional source of coupling loss between onchip components as the wave packet spreads laterally during propagation. To address this issue, plasmonic Airy beams (PABs) have recently been introduced [14-17]. The PAB is a solution of the one-dimensional free-particle Schrödinger-like equation, to which the two-dimensional Helmholtz equation reduces in the slowly varying envelope approximation that is for small variations of the transverse SPP profile along the propagation distance [14]. PABs propagate without spreading for a finite distance, but their trajectory bends, severely limiting possible device applications. This lack of symmetry in the transverse field distribution prompted us to investigate SPPs propagating without diffraction and in a straight line. A first step in this direction has been theoretically proposed in Ref. [18] by considering the propagation of high order plasmon modes at the interfaces of multilayered metal-dielectric superlattices. Although this approach is experimentally cumbersome, it proposes a SPP beam which propagates in a straight rather than parabolic trajectory, like PAB. Here we demonstrate both experimentally and theoretically that a new surface wave, with similar properties, can be generated at a single interface with simple metallic gratings.

For monochromatic SPPs, the electric field component $E_{z, d}$ (in the dielectric half-space) normal to the interface satisfies the equation

$$
\nabla^{2} E_{z}+\varepsilon_{d} k_{0}^{2} E_{z}=0,
$$

where $k_{0}=\omega / c$ is the wave vector in vacuum and $\varepsilon_{d}$ is the permittivity of the dielectric. We have dropped for simplicity of notation the subscript $d$. Note that the other field components can be derived from the knowledge of $E_{z}$ [19]. We seek a surface wave solution of Eq. (1) in the form $E_{z}=A(x, y) \exp (-\alpha z)$, where the exponential accounts for the evanescent wave decay in the dielectric, characteristic of waves propagating at an interface such as SPPs. Note that the expression for $E_{z}$ describes the field distribution in the dielectric half-space $z>0$ and that a similar expression with a different confinement factor $\alpha$ can be written for $z<0$. One can show that

$$
E_{z}=A \exp \left(j k_{x} x\right) \cos \left(k_{y} y\right) \exp (-\alpha z)
$$


satisfies Eq. (1), where $A$ is a complex constant. By using the continuity of the normal component of the $D$ vector and the parallel components of the $E$ vector at the interface, the following dispersion relation can be obtained [19]: $k_{\mathrm{sp}}^{2}=$ $k_{x}^{2}+k_{y}^{2}=\alpha^{2}+\varepsilon_{d} k_{0}^{2}=k_{0}^{2} \varepsilon_{d} \varepsilon_{m} /\left(\varepsilon_{d}+\varepsilon_{m}\right)$, where $k_{x}$ and $k_{y}$ are the $x$ and $y$ components, respectively, of the propagating SPP wave vector $k_{\mathrm{sp}}$, which is a complex quantity to account for SPP attenuation. Here, $\varepsilon_{m}$ is the complex permittivity of the metal, while $\varepsilon_{d}$ is real, because the dielectric is considered to be transparent at optical wavelengths of interest. In our experiment, the dielectric is air.

Similar to the case of free-space Bessel beams, in which the field can be decomposed into plane wave components with wave vectors on a cone [3], an ideal cosine beam described by Eq. (2) can be decomposed into two SPP plane waves propagating with the half angle in between defined by $\theta=\sin ^{-1}\left(k_{y} / k_{\mathrm{sp}}{ }^{\prime}\right)$, where $k_{\mathrm{sp}}{ }^{\prime}$ is the real part of $k_{\mathrm{sp}}$. This decomposition suggests a direct way to create a source of cosine beams [Fig. 1(a)]. In the limit of a small angle, the solution represents surface waves propagating in the $x$ direction without diffraction, i.e., with a transverse intensity profile independent of $x$. This beam can be regarded as the two-dimensional counterpart of a nondiffracting Bessel beam in free space.

It is important to note that an ideal Bessel beam solution carries infinite energy [3], because it is not squareintegrable and therefore cannot be realized in practice. It is nevertheless possible to experimentally approach the ideal Bessel solution by modulating it with a Gaussian envelope. The resulting Bessel-Gauss beam preserves nondiffracting properties in the paraxial approximation while carrying a finite energy [20]. The nondiffracting
SPP solution presented in Eq. (2) suffers from the same problem as the original Bessel beam solution. We therefore introduce the cosine-Gauss beam (CGB), a SPP characterized by

$$
E_{z}=A \exp \left(j k_{x} x\right) \cos \left(k_{y} y\right) \exp \left(-\frac{y^{2}}{w_{0}^{2}}\right) \exp (-\alpha z)
$$

where $w_{0}$ denotes the beam waist (Supplemental Fig. 1 [21]). Our study of the propagation of the CGB shows that it provides a good paraxial approximation to the nondiffracting solution, in a way similar to free-space BesselGauss beams. Assume that along the interface $(z=0)$ the field distribution at $x=0$ is a CGB: $E_{z}\left(0, y^{\prime}\right)=$ $A \cos \left(k_{y} y^{\prime}\right) \exp \left(-y \prime^{2} / w_{0}^{2}\right)$. A calculation of the propagation integral [22] shows that, in the paraxial approximation, the field maintains the same CGB transverse profile regardless of the propagation distance $x$ :

$$
E_{z}(x, y) \cong A^{\prime} f(x) \exp \left(-\frac{y^{2}}{w_{0}^{2}}\right) \cos \left(k_{y} y\right)
$$

where $f(x)$ depends on $k_{\mathrm{sp}}, w_{0}$, and $k_{y}$ (Supplemental Material, Part A [21]). From Eq. (4), we conclude that the CGB represents a nondiffracting surface wave of finite energy.

A straightforward way, depicted in Fig. 1(a), of generating nondiffracting SPPs is to use line sources represented by gratings made of grooves of length $2 D$ defined in the metal, which under normal illumination generate SPP plane waves propagating normal to the grooves. The assumption of plane waves is an excellent approximation when $D$ is much larger than the surface plasmon wavelength. The cosine beam forms in front of the coupling (a)

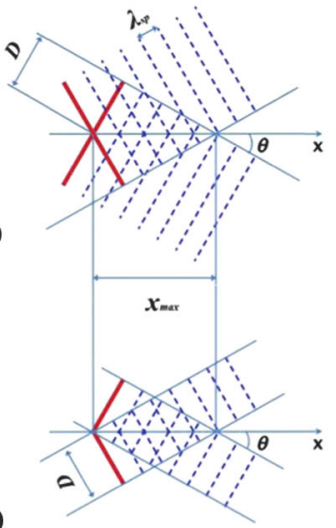

(c)

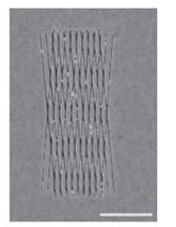

(d)



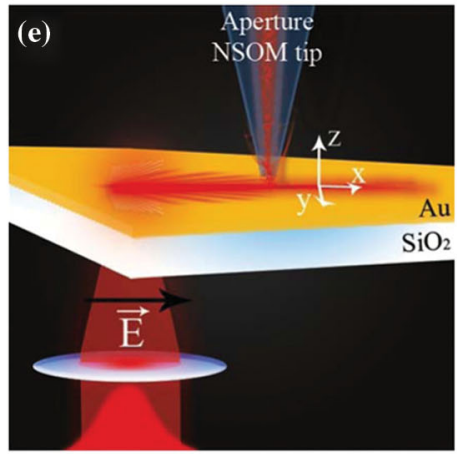

FIG. 1 (color). Generation of nondiffracting SPPs. Methods used to generate (a) the CGB and (b) the LCGB. Scheme (a) by symmetry launches bidirectional CGBs, when illuminated, while scheme (b) is a one-directional launcher of LCGBs. Each pair of red lines represents grooves $\left(D \sim 10 \mu \mathrm{m} ; \theta \sim 5^{\circ}\right)$ that are part of metallic gratings sculpted on an Au film deposited on a glass substrate, as illustrated in the scanning electron micrographs (c),(d). SPPs are excited at normal incidence by a focused Gaussian beam from a laser $(\lambda \sim 0.74 \mu \mathrm{m})$ polarized parallel to the $x$ axis. The SPP plane waves of equal amplitude launched by the pair of gratings interfere constructively to form nondiffracting surface waves for a propagation distance $x_{\max }=D / \sin \theta \sim 120 \mu \mathrm{m}$. The period of the gratings is designed to match the wavelength of SPPs, so that the latter can be resonantly excited by vertical illumination. Scheme (b) gives a SPP beam more localized in the transverse direction than that in (a). (e) Experimental setup. The SPP field distribution is measured by an apertured NSOM. 
grooves, in the area where the two SPP plane waves overlap [Fig. 1(a)]. The maximum distance within which the generated beam remains nondiffracting can be estimated geometrically from Fig. 1(a) by using $x_{\max }=D / \sin \theta$. The two plane waves constructively interfere over this distance, giving rise to a standing wave of profile $\cos \left(k_{y} y\right)$ in the $y$ direction. The paraxial regime of these propagating waves allows one to achieve very long nondiffracting propagation distances $x_{\max }$. To generate the CGB, the amplitudes of the plane waves launched by the two line sources need to be modulated by a Gaussian profile. This is accomplished in our experiments by illumination with a normal incidence Gaussian beam of waist $w_{0}<D ; w_{0}$ then replaces $D$ in the expression for $x_{\max }$.

Under these assumptions, the field distribution right after the plasmonic couplers, that is, at $\left(x=0, y^{\prime}\right)$, can be modeled by

$$
\begin{aligned}
E_{z}\left(0, y^{\prime}\right)= & \frac{A}{2}\left[\exp \left(-j y^{\prime} k_{\mathrm{sp}}^{\prime} \sin \theta\right)+\exp \left(j y^{\prime} k_{\mathrm{sp}}^{\prime} \sin \theta\right)\right] \\
& \times \exp \left(-\frac{y^{\prime 2}}{w_{0}^{2}}\right) \\
= & A \cos \left(y^{\prime} k_{\mathrm{sp}}^{\prime} \sin \theta\right) \exp \left(-\frac{y \prime^{2}}{w_{0}^{2}}\right) .
\end{aligned}
$$

Thus the device configuration of Fig. 1(a) generates a CGB. This transverse profile is maintained as the field propagates along $x$ (Supplemental Material, Part A [21]).

A numerical calculation [using Eq. (A1)] also confirmed that the transverse intensity profile remains unchanged (Supplemental Fig. 2 [21]) within the propagation length, which is associated with absorption losses (estimated $\sim 30 \mu \mathrm{m}$ for an air/Au interface at $740 \mathrm{~nm}$ ). We notice that, from the simple geometrical picture of Fig. 1(a), half of either line source radiates fields away from the propagation axis. A design modification that gives increased transverse localization, i.e., with less intensity in the side lobes, is shown in Fig. 1(b) and was found to exhibit nondiffracting properties as well. The field generated immediately to the right of the device $(x=0)$ in Fig. 1(b) can be expressed as $E_{z}\left(0, y^{\prime}\right)=A \exp \left(-j\left|y^{\prime}\right| k_{\mathrm{sp}}^{\prime} \sin \theta\right) \times$ $\exp \left(-y^{12} / w_{0}^{2}\right)$. Using Eq. (A2), one finds that the field profile $E_{z}(x, y)$ [Eq. (B3)] is no longer a diffraction-free solution of the wave equation. However, numerical simulations in Supplemental Fig. 3 [21] (also confirmed by analytical calculations in Part B of Supplemental Material) show that the central part of the field profile is well described by a cosine-Gaussian function [Eq. (B7)] in accordance with our SPP imaging experiments, which demonstrate that the beam width is independent of propagation distance up to $\sim 80 \mu \mathrm{m}$.

The advantage of this SPP, which we shall call localized cosine-Gauss beam (LCGB), for applications is the increased transverse confinement compared to the CGB, while maintaining negligible diffraction. One can in fact show that the device of Fig. 1(b) can generate LCGBs with axial intensities much larger than conventional SPP beams launched by a single grating of comparable transverse dimension.

In the following, we study the proposed nondiffracting SPPs by performing the experiment with the setup depicted in Fig. 1(e). The device used to generate the nondiffracting SPPs comprises two identical gold gratings that convert part of the incident free-space light into propagating SPPs. The configuration [Fig. 1(c) or 1(d)] of the two gratings will determine which type of nondiffracting SPPs will be generated. The Gaussian amplitude profile required by the CGB was realized by illuminating the gratings with a $\mathrm{TEM}_{00}$ fundamental mode of a laser focused from the substrate side. We then mapped the two-dimensional SPP field distribution by collecting the in-plane field components using a near-field scanning optical microscope (NSOM) working in aperture mode [23] with a broadband illumination combined with hyperspectral detection [24] (see details in Supplemental Material [21]). The high quality near-field images of the propagating SPPs enable us to extract the transverse intensity profile of the CGBs for a comparison with both the analytical calculation and the finite-difference time-domain (FDTD) method. In our experiment, the incident wavelength is $\lambda=740 \mathrm{~nm}$, and the other parameters are $w_{0}=8 \mu \mathrm{m}$ and $\theta=5^{\circ}$ [Figs. 1(a) and 1(b)]. For gold at this wavelength the real part of $k_{\mathrm{sp}}$ is $k_{\mathrm{sp}}^{\prime}=8.7 \times 10^{6} \mathrm{~m}^{-1}$, and the imaginary part is $k_{\mathrm{sp}}^{\prime \prime}=$ $1.7 \times 10^{4} \mathrm{~m}^{-1}$. We designed our devices with a small $\theta$ to enhance the maximum nondiffracting distance $x_{\max }$.

Figures 2 and 3 show that, for the beams generated by the two devices, the full-wave calculations and the experimental results are in good agreement with theoretical predictions within the maximum nondiffracting distance. In Fig. 4, we further studied the beam width of the two types of nondiffracting waves as a function of the angle between the SPP generating gratings and propagation distance. The results indicate that the transverse size of the main lobe decreases with increasing angle, which offers considerable design flexibility. As shown in Fig. 4(b), the second device design [Figs. 1(b) and 1(d)] with $\theta=5^{\circ}$ leads to a nondiffracting distance as long as $80 \mu \mathrm{m}$, which is considerably longer than the reported propagation length of PABs [15-17] after accounting for the wavelength and beam width difference.

In conclusion, we introduced the cosine-Gauss beam, a nondiffracting surface wave solution of the wave equation in the paraxial regime, and we experimentally proved its properties by mapping the near-field distribution. The double-grating devices demonstrated in this work represent an efficient and straightforward way to generate a localized SPP beam with minimum diffraction. It is worth pointing out that, as opposed to optical solitons which can also propagate conserving the same transverse profile during propagation [1], our diffraction-free functions are linear solutions of the propagation equation. In this linear regime, we nevertheless have to stress that diffraction, as an inevitable effect of wave propagation, still exists for any 

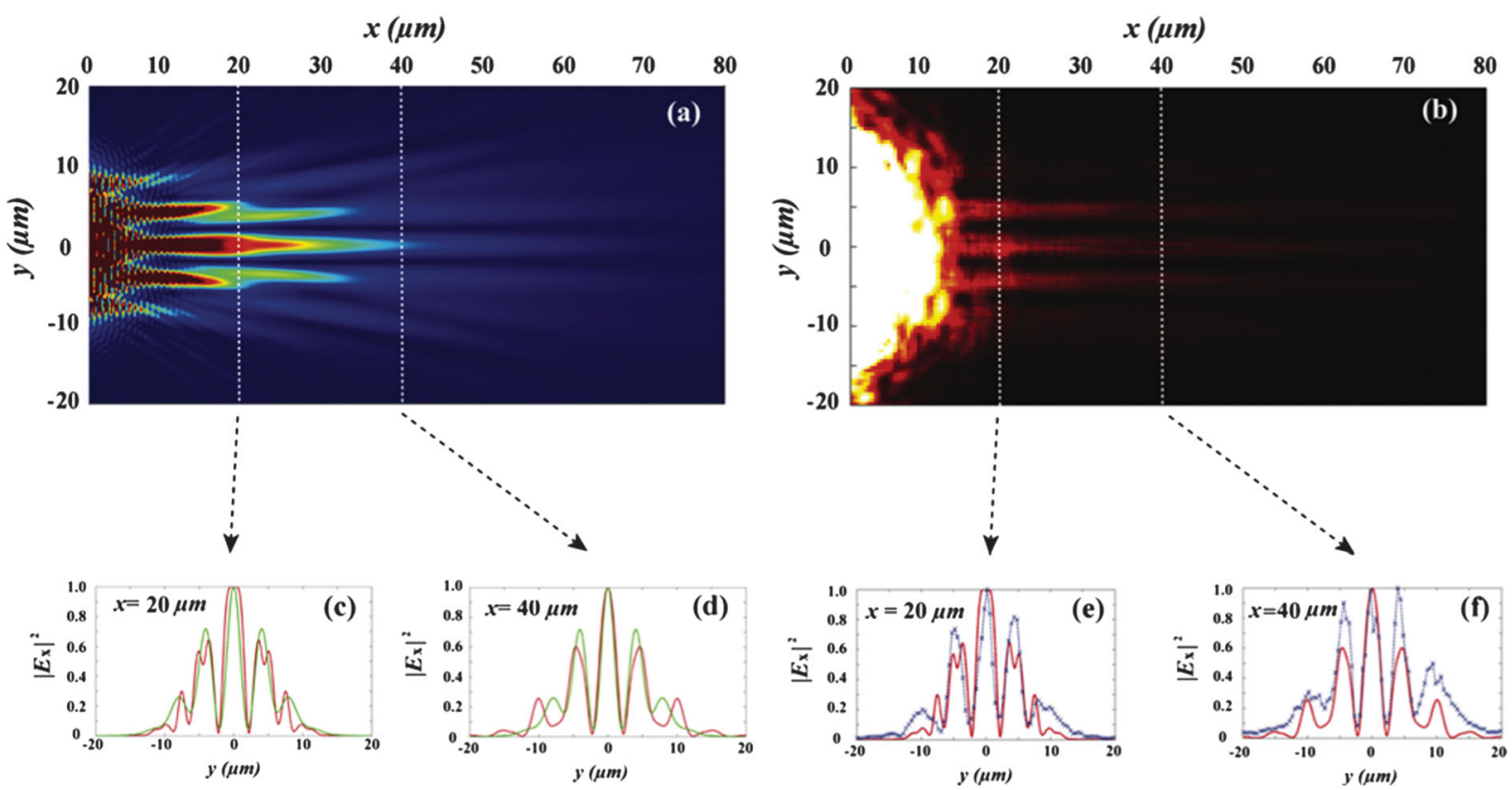

FIG. 2 (color). CGB generated by two overlapped coupling gratings as shown in Fig. 1(c). The near-field intensity distributions (inplane components) obtained from (a) FDTD simulations and (b) experimentally using a NSOM. (c)-(f) Transverse intensity distributions at specific propagation distances $x$ [green curve, analytical calculations; red curve, simulations by FDTD; blue curve, intensity line scans obtained from the NSOM image in (b)]. Note that it takes a distance of several microns to form the nondiffracting beam, which can be understood by observing that the latter is fully formed only after the surface plasmon polaritons launched by the grooves of the two gratings have sufficiently overlapped Fig. 1(a). Similar considerations apply to a localized cosine-Gaussian beam (Fig. 3)

nondiffracting beams that carry finite energy. In our case it leads to a maximum nondiffracting distance. Physically, the nondiffracting property of our plasmonic beams can be attributed to the large (as compared to the narrow main lobe) transverse dimension of the total field including side lobes. However, the nondiffracting plasmonic beams do provide an invaluable approach to realizing a more confined local field (narrow main lobe) while maintaining the small divergence that is usually associated with a more spread-out transverse field. The CGB and the Airy beam
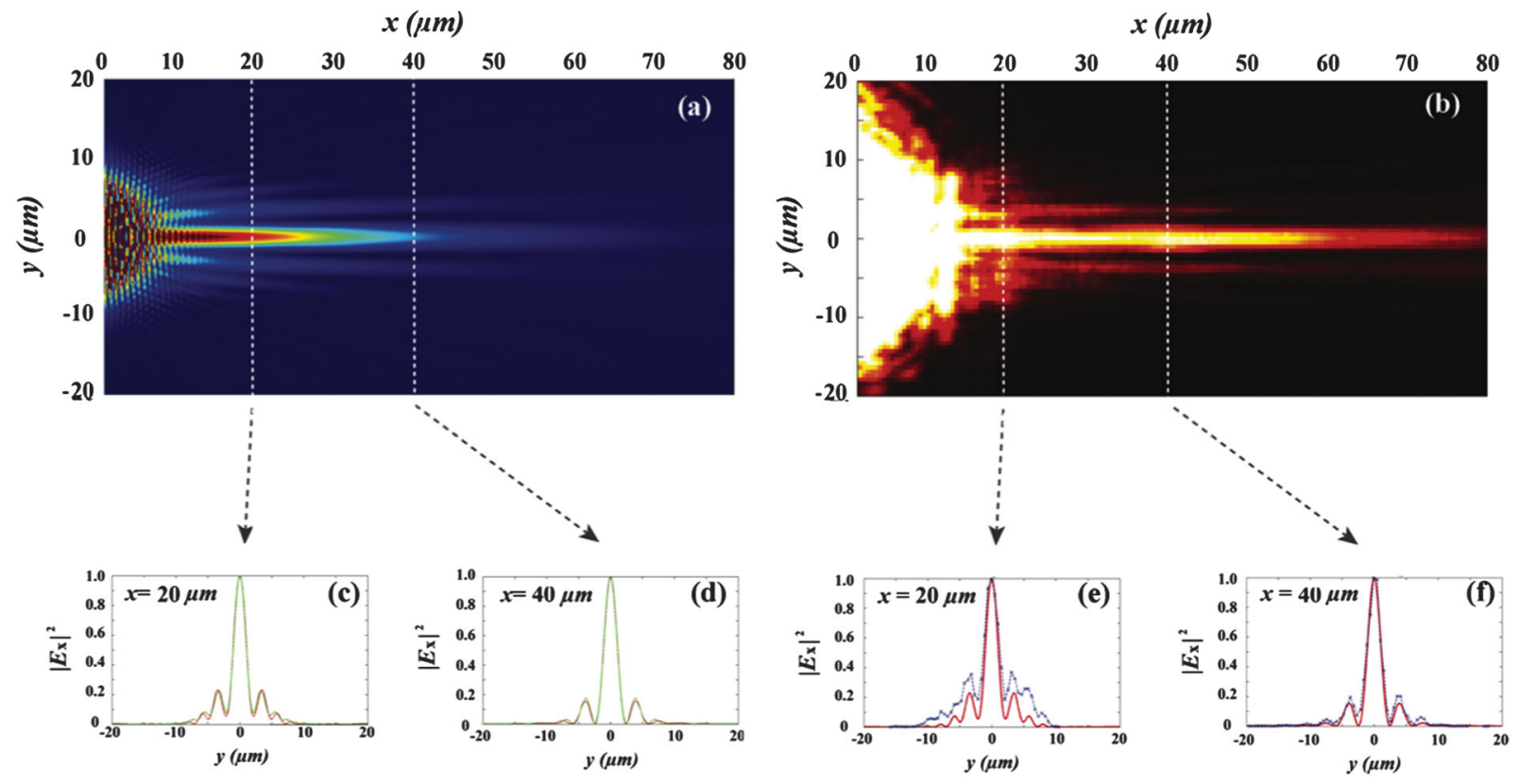

FIG. 3 (color). The LCGB generated by two gratings at an angle as shown in Fig. 1(d). The near-field intensity distributions (in-plane components) obtained (a) by using FDTD simulations and (b) experimentally with an NSOM. (c)-(f) Transverse intensity distributions at specific propagation distances [green curve, analytical calculations; red curve, simulations by FDTD; blue curve, intensity line scans obtained from the NSOM image (b)]. 

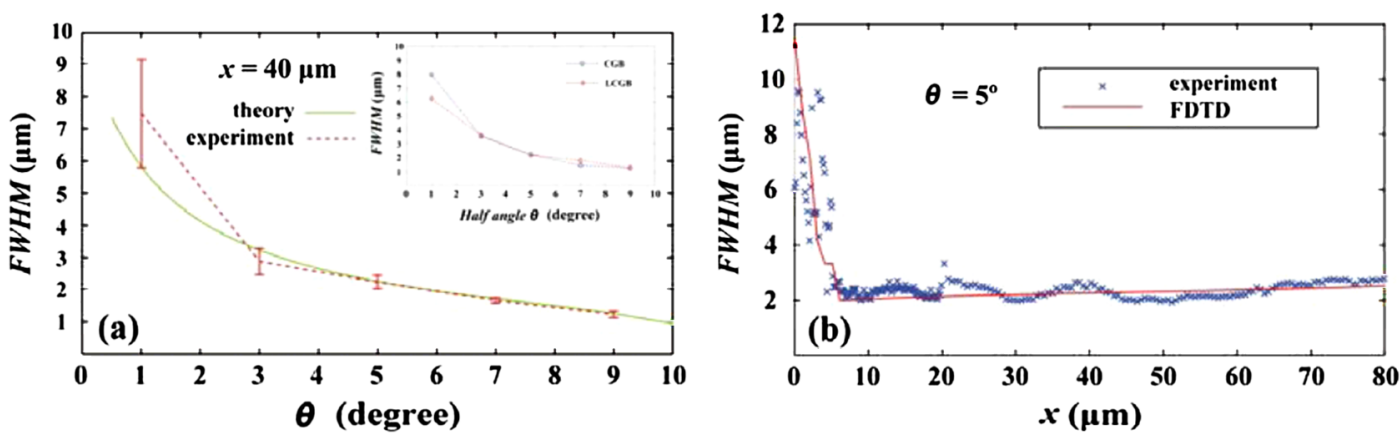

FIG. 4 (color online). (a) The FWHM of the main lobe of the LCGB at the propagation distance $x=40 \mu \mathrm{m}$ as a function of the half angle $\theta$, which defines the relative orientation of the gratings. The inset shows that the two types of nondiffracting SPPs have comparable width. (b) FWHM of the main lobe of the LCGB versus propagation distance. Experimental data (blue cross) and FDTD simulations (red solid line) are compared for $\theta=5^{\circ}$. The initial drop in beam width is related to the finite distance required to form the nondiffracting beam.

result from the interference of different types of waves leading to different trajectories. The nonstraight trajectory of Airy beams is attributed to the caustic of the secondary waves, which creates a curved envelope of intense light [25]. The straight propagation of the CGB simply results from constructive interference of two tilted plane waves propagating in phase along the symmetry axis. Because of their unique properties, CGB surface waves could find potential applications in areas such as near-field plasmonic manipulation of particles and the transmission of plasmonic signals among on-chip devices.

J.L. and J.D. contributed equally to this work. The Harvard University authors acknowledge partial support from the Air Force Office of Scientific Research under AFOSR Contract No. FA9550-09-1-0505. J. L. also acknowledges the support from the Agency for Science, Technology and Research (A*STAR), Singapore and P. G. partial funding from the Robert A. Welch Foundation (A-1261). Device fabrication was carried out at the Center for Nanoscale Systems (CNS) at Harvard University. Harvard CNS is a member of the National Nanotechnology Infrastructure Network (NNIN). J.D., B.C., and F.d.F. acknowledge the Burgundi Regional Council from France for financial support through the PHOTCOM project.

*Corresponding author. capasso@seas.harvard.edu

[1] G. I. Stegeman and M. Segev, Science 286, 1518 (1999).

[2] C. J. R. Sheppard and T. Wilson, MOA 2, 105 (1978).

[3] J. Durnin, J. J. Miceli, Jr., and J. H. Eberly, Phys. Rev. Lett. 58, 1499 (1987).

[4] J. C. Gutiérrez-Vega, M. D. Iturbe-Castillo, and S. ChávezCerda, Opt. Lett. 25, 1493 (2000).

[5] M. A. Bandres, J.C. Gutiérrez-Vega, and S. ChávezCerda, Opt. Lett. 29, 44 (2004).

[6] G. A. Siviloglou, J. Broky, A. Dogariu, and D. N. Christodoulides, Phys. Rev. Lett. 99, 213901 (2007).
[7] I. Kaminer, R. Bekenstein, J. Nemirovsky, and M. Segev, Phys. Rev. Lett. 108, 163901 (2012).

[8] W. L. Barnes, A. Dereux, and T. W. Ebbesen, Nature (London) 424, 824 (2003).

[9] E. Ozbay, Science 311, 189 (2006).

[10] M. L. Brongersma, J. A. Schuller, J. White, Y. C. Jun, S. I. Bozhevolnyi, T. Sondergaard, and R. Zia, in Plasmonic Nanoguides and Circuits, edited by S. I. Bozhevolnyi (World Scientific, Singapore, 2009).

[11] D.F.P. Pile and D. K. Gramotnev, Opt. Lett. 29, 1069 (2004).

[12] P. Berini, R. Charbonneau, N. Lahoud, and G. Mattiussi, J. Appl. Phys. 98, 043109 (2005).

[13] S. I. Bozhevolnyi, V. S. Volkov, E. Devaux, J.-Y. Laluet, and T. W. Ebbesen, Nature (London) 440, 508 (2006).

[14] A. Salandrino and D. N. Christodoulides, Opt. Lett. 35, 2082 (2010).

[15] A. Minovich, A. E. Klein, N. Janunts, T. Pertsch, D. N. Neshev, and Y.S. Kivshar, Phys. Rev. Lett. 107, 116802 (2011).

[16] L. Li, T. Li, S. M. Wang, C. Zhang, and S. N. Zhu, Phys. Rev. Lett. 107, 126804 (2011).

[17] P. Zhang, S. Wang, Y. Liu, X. Yin, C. Lu, Z. Chen, and X. Zhang, Opt. Lett. 36, 3191 (2011).

[18] C. J. Zapata-Rodriguez, S. V. Milivoj, R. Belic, D. Pastor, and J. J. Miret, Opt. Express 19, 19572 (2011).

[19] S. A. Maier, Plasmonics: Fundamentals and Applications (Springer, New York, 2007).

[20] F. Gori, G. Guattari, and C. Padovani, Opt. Commun. 64, 491 (1987).

[21] See Supplemental Material at http://link.aps.org/ supplemental/10.1103/PhysRevLett.109.093904 for detailed calculations and self-healing properties of the CGB.

[22] T. V. Teperik, A. Archambault, F. Marquier, and J.J. Greffet, Opt. Express 17, 17483 (2009).

[23] L. Lalouat, B. Cluzel, C. Dumas, L. Salomon, and F. de Fornel, Phys. Rev. B 83, 115326 (2011).

[24] J.-S. Bouillard, S. Vilain, W. Dickson, and A. Zayats, Opt. Express 18, 16513 (2010).

[25] L. Froehly, F. Courvoisier, A. Mathis, M. Jacquot, L. Furfaro, R. Giust, P. A. Lacourt, and J. M. Dudley, Opt. Express 19, 16455 (2011). 